\title{
Design methodology for hybrid (VTOL + Fixed Wing) unmanned aerial vehicles
}

\begin{abstract}
The evolution in the missions' complexity of both military aircraft and Unmanned Aerial Vehicles (UAVs) and the increase in civilian air-traffic, with limited runways, have led to the development of a new category of aircraft called Transitional Aircraft (TA). The goal of TA is to take advantages of both the characteristics of fixed-wing aircraft, like high speed, range and endurance, and of rotorcraft such as hovering, low-speed flight and Vertical Take-off and Landing (VTOL). Despite the abundant knowledge on designing both fixed-wing aircraft and rotorcraft, designing TA has proven to be a challenging process and poorly understood. Currently there is no well accepted generic methodology that can be used for designing this type of aircraft such as tiltrotor systems due to the fact that there are numerous challenges regarding the selection of the proper TA configuration. Although somewhat effective, previously developed TA suffer from problems regarding the mutual aerodynamic interaction that occurs during the transition flight phase when the aircraft transitions between fixed-wing and rotorcraft flight modes. In order to cope with these problems, this paper presents a preliminary design methodology for TA with a set of newly developed mathematical formulations that integrate traditional fixed-wing and rotorcraft aircraft design methodologies into a comprehensive TA design methodology. Due to the potential large set of TA configurations that exist and can be proposed, this paper focuses on transitional tiltrotors which have been identified as critical importance. The proposed TA design approach is used to analyze a hypothetical TA configuration under development by the authors of this paper showing that the proposed design approach can be used to prevent some of the frequent shortcomings found on previously developed aircraft. The methodology is able to assist TA designers obtain adequate power, wing, and rotor disc loadings using the developed sizing mathematical formulations for tiltrotor aircraft based on integrating the performance requirements in the fixed-wing, rotorcraft, and transition flying modes.
\end{abstract}

Volume 2 Issue 3 - 2018

\author{
Ashraf M Kamal,' Alex Ramirez-Serrano ${ }^{2}$ \\ 'Ph D Student, Department of Mechanical \& Manufacturing \\ Engineering, University of Calgary, Canada \\ ${ }^{2}$ Professor and Director Unmanned Vehicle Robotics R \& D \\ Lab, Department of Mechanical \& Manufacturing Engineering, \\ University of Calgary, Canada
}

Correspondence: Alex Ramirez-Serrano, Professor and Director Unmanned Vehicle Robotics R\&D Lab, Department of Mechanical \& Manufacturing Engineering, University of Calgary, 2500 Univ. Drive NW., Calgary, Canada,Tel +I (403) 220-3632, Email aramirez@ucalgary.ca

Received: February 16, 2018 | Published: June 06, 2018

\section{Nomenclature}

$$
\begin{array}{ll}
A & =\text { rotor disk area of rotorcraft, } m^{2} \\
A R & =\text { wing aspect ratio } \\
C_{d} & =\text { average blade drag coefficient } \\
C_{D o} & =\text { aircraft zero lift drag coefficient }
\end{array}
$$

$P_{i}, P_{c}, P_{o}, P_{p}=\quad$ induced, climb, blades' profile and parasite power required for the rotorcraft respectively, Watt

$$
\begin{array}{ll}
S & =\text { wing area, } \mathrm{m}^{2} \\
V_{s} & =\text { stall speed, } \mathrm{m} / \mathrm{s} \\
V_{y} & =\text { required rate of climb, } \mathrm{m} / \mathrm{s} \\
V_{y . c} & =\text { rate of climb at service ceiling, } \mathrm{m} / \mathrm{s} \\
v_{i} & =\text { induced velocity at rotor disc, } \mathrm{m} / \mathrm{s} \\
W & =\text { aircraft weight, } N \\
W_{\text {Empty }} & =\text { aircraft empty weight, } N \\
W_{T O} & =\text { aircraft maximum take-off weight, } N \\
\eta_{p} & =\text { propeller efficiency } \\
\rho_{o} & =\text { air density at sea level altitude, } \mathrm{kg} / \mathrm{m}^{3} \\
\rho_{C} & =\text { air density at service ceiling altitude, } \mathrm{kg} / \mathrm{m}^{3}
\end{array}
$$

$$
\begin{aligned}
& \begin{array}{l}
\sigma \\
\sigma
\end{array} \quad \text { main rotor solidity ratio } \\
& \begin{array}{l}
\sigma_{\text {air }} \quad=\text { relative air density/de-rating factor for reciprocating } \\
\text { engine/electric motors }
\end{array} \\
& \begin{array}{l}
\mu \quad=\text { forward dimensionless velocity in rotor disc plane } \\
(\text { advance ratio) }
\end{array} \\
& \mu_{f} \quad=\text { run way friction coefficient }
\end{aligned}
$$

\section{Introduction}

Transitional aircraft are systems capable of flying as fixed-wing aircraft or rotorcraft as well as transition between these modes when desired/needed. Operation in helicopter mode makes the aircraft possible to Vertical Take-off and Landing (VTOL), fly at low speeds in any direction (e.g., forward and sideways), hover and perform some difficult maneuvers. In the fixed-wing mode, due to stall problems on the lifting surfaces, low speeds are not possible. However, the aircraft can make a substantial increase in range, endurance, altitude, payload carrying capacity, and maximum forward speed compared to typical rotorcraft. Therefore, Transitional Aircraft (TA) extend the aircraft flight envelope, mission, and performance of a typical aircraft by incorporating the characteristics of both helicopters and fixed-wing airplanes. ${ }^{1-3}$ Throughout the last decades, numerous attempts have been devoted in developing TA. However, only a limited number of successful aircraft have been realized and relatively successful systems still present numerous challenges as they do not operate optimally. ${ }^{4}$ Diverse concepts have been examined/developed in previous works in an attempt to find better designs. ${ }^{5}$ The most 
common and successful concepts are tiltrotors, tilt-wings, and tailsitter (Figure 1). These concepts use the same propulsion system in both helicopter and airplane modes by either tilting the population system alone (e.g., tiltrotors) or with the wing (e.g., tilt-wing) or with the fuselage (e.g., tail sitter). However, other simplistic designs exist that take traditional rotorcraft (e.g., helicopters and quadrotor UAV) and extend their capabilities by incorporating additional (push/pull) propeller systems (examples shown in Figure 2). Although somewhat effective, these designs are far from optimal and improved theories as well as specific design methodologies for optimal/refined TA are needed.



Rotormast V-22 tiltrotor UAV 6

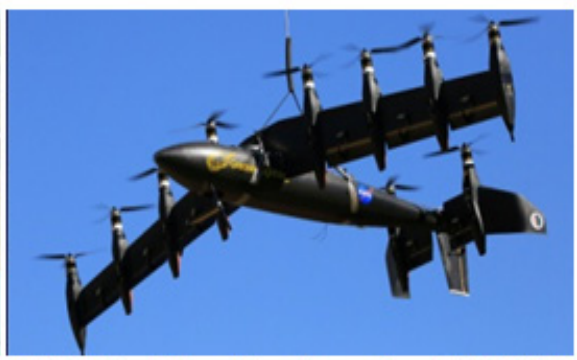

NASA GL-10 tilt-wing UAV ${ }^{7}$



Quantix tail sitter UAV

Figure I Examples of tiltrotor, tilt-wing, and tail sitter UAVs.

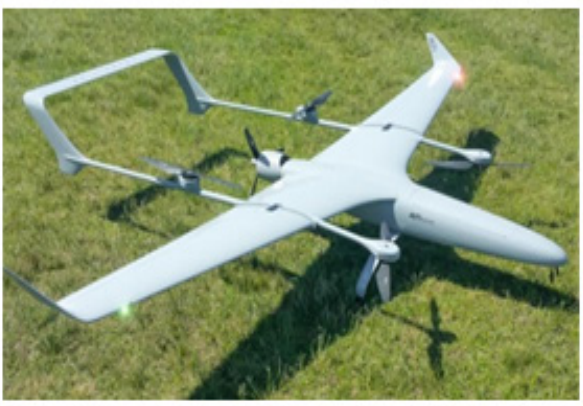

ALTI Transition ${ }^{9}$

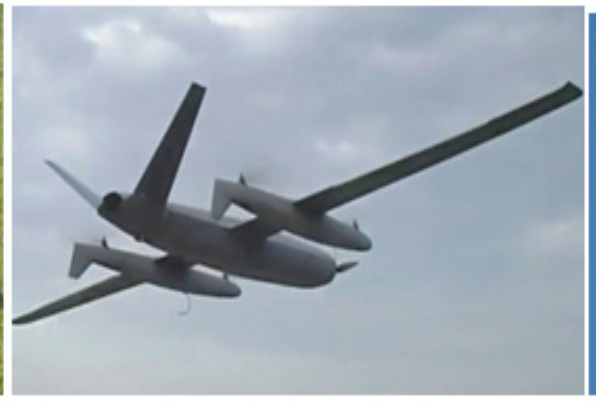

Dronetech's AV-1 Albatross ${ }^{10}$



Arcturus JUMP $20^{11}$

Figure 2 Simplistic designs of transitional quadcopters.

The limited available studies on TA have focused on investigating/ analyzing the mutual aerodynamic interaction between the propulsion system (e.g., propellers' downwash) and the aircraft's parts (e.g., lifting surfaces), which has provided the means to proposing new aircraft configurations, and designing the corresponding flight controllers. Despite the importance and the need for developing TA, the design problems associated with such type of aircraft has hitherto received very little attention in the research literature. Even though most previously developed aircraft are unstable, ${ }^{12-14}$ no attention has been paid to develop/discuss methodologies to design stable TA. This paper presents the first step in the design of TA, TA preliminary design, and some of the challenges accompanying this process. The ultimate goal of the proposed work is on developing a formal preliminary design approach for TA, with the aim to reduce transition time, enhance stable transition, and reduce dead weight for any specific flight mode. That is, the aim is to use a single aircraft configuration that employs the same components in all of the three flight phases (i.e., VTOL, transition, and cruise). In this paper, "dead weight" is defined as components and systems that are only used in one of the three flight modes present in TA. Thus, reducing/eliminating dead weight is desirable.

In the context of conventional fixed-wing aircraft, preliminary design includes the estimation of the engine power/thrust and wing loadings. This phase is one of the most important design steps for developing aircraft. In the context of TA design, the vast majority of studies and developments have focused on presenting new configurations using either traditional design methods for fixed-wing aircraft or historical data, ${ }^{12,15-17}$ rather than looking into the importance of the preliminary design method itself. For instance, averaged historical data for manned aircraft and fixed-wing stall speed limit have been used to estimate the required power and wing loadings respectively for the design of a tilt-ducts $\mathrm{UAV} .{ }^{12}$ Additionally, the rotor diameter has been selected based on fixed-wing aircraft methods and the sonic speed limit. Despite having good fixed-wing flight performance, the developed $\mathrm{UAV}^{12}$ was found to be unstable in both hover and transition flight modes. Although historical data for the same aircraft category, type, and size, may be used in the early design stages, ${ }^{16,18}$ the used data have come from different aircraft category, type, and size mainly due to limited available data for TA. ${ }^{12}$

Even though previously developed techniques have shown to be somewhat useful, using such approaches are far from optimal. On the other hand, some references, ${ }^{16}$ suggested to use thrust over weight ratio between 1.2 and 1.5 for VTOL with good results. Such approach has been used in developing tail-sitter UAVs. ${ }^{19}$ Another approach has suggested to use a traditional fixed-wing aircraft design methodology 
with subsequent enforcement of a power/thrust loading constraint for the VTOL mode. ${ }^{17}$ Such suggestion is based on historical databases to set the minimum required power/thrust. Such databases have been developed based on manned VTOL aircraft classified according to using the propulsion system in generating lift and thrust, without considering the aircraft type, weight, mission, and category. ${ }^{17}$ Although using historical data has been shown to be practically possible, this may lead to having unneeded excess power, which penalizes the design by increasing the total cost, required structural integrity, and weight with the associated reduction in aircraft performance and flying characteristics.

In contrast to using historical data, other studies have proposed to use momentum theory to calculate the power loading in designing tiltwing and other transitional type of aircraft. The first study ${ }^{20}$ designed and tested a tilt-wing UAV, whereas the others to maintain flight ${ }^{13,14}$ did the same process for a transitional UAV with two tilt-motors in the front, and a fixed coaxial ducted fan in the rear. Unfortunately, the later UAV is also inherently unstable and when in operation, it heavily relies on the flight control system. ${ }^{13,14}$ Although momentum theory is considered better than historical data in estimating the power, it can only be used to calculate the required power during the hovering mode, which is thought to be not sufficient for all the three flight phases in a typical TA mission. Based on previous work, which has shown that it is possible to fly transitional tiltrotor aircraft in both modes (VTOL and fixed-wing) with only one aircraft configuration. Herein, we focus on the design of such aircraft with added lift surfaces, where most of the aircraft sub-systems are used in both flying modes. Thus reducing the dead weight that has been typically used/added to the aircraft in any flying mode.

\section{Problem definition}

Obtaining stable and smooth transition in minimum time is one of the important requirements for TA, thus minimizing any operational losses that might be encountered in the transition phase. Losing altitude during transition is one of the potential sources of instability that leads to a somewhat rough transition. Although it is not clearly discussed in the literature, it is thought that proper sizing of TA, specifically sizing of engine power, plays a very important role in obtaining effective transition flight while preserving the aircraft's altitude. During transition of tiltrotor aircraft, the aircraft's propulsion system (i.e., rotors) is tilted, so the engine power should be sized properly to allow generate more thrust to carry the aircraft's weight, and provide the necessary thrust for forward acceleration to gain forward speed. This will allow preserving the aircraft's altitude and reaching the desired wing-borne speed rapidly, which consequently should decrease the required transition time. If the aircraft's engine is sized according to the fixed-wing flight mode only, the engine power either will be insufficient for performing the transition operation or may perform the transition with losing altitude. In such case, the time needed to reach the wing-borne speed is increased. Under such conditions, the aircraft will lose altitude, stability and the transition time will increase resulting in performance deficiencies. An alternative easy, but not optimal, solution is to use higher engine power. For these reasons, including the helicopter and transition flight modes in the initial sizing of TA is important to obtain the appropriate size of engine, wing and rotor.

Due to the diverse complexities associated with diverse TA, this paper focuses on transitional UAVs that use the same propulsion system for both fixed-wing and helicopter flying modes, which is yet one of the most challenging configurations. As most of the available UAVs use propeller-driven propulsion system, this paper will consider only tiltrotor UAVs with added lifting surfaces.

Thus the problem statement of this paper is:

Develop a preliminary design methodology for tiltrotor aircraft that would enable them to transition from VTOL to fixed-wing flight mode by reducing transition time, enhance stable transition and reduce dead weight for any specific flight mode while enabling close to optimal flight (VTOL, Transition and fixed-wing) which maximizes aircraft systems usage in all flying modes.

Due to the high complexities associated with the design of TA and the fact that the transition from VTOL to fixed-wing is of higher complexity compared to transition from fixed-wing to VTOL, the following assumption is considered: i) it is assumed that the air density during transition does not change. That is, it is assumed that the altitude of the aircraft remains constant and that the range of speed is not large enough to have a change in air density surrounding the aircraft during the transition phase. The reason for the transition from VTOL to fixed-wing being of higher complexity this is that the power required during transition from VTOL to fixed-wing is higher than the power required during transition from fixed-wing to VTOL.

Furthermore, due to potential large set of TA configurations and the scant available literature in the area and in order to further simplify the complexity of the problem at hand, some constraints are put in place when developing the proposed solution:

a. The work reported in this paper is devoted to tiltrotor aircraft that use the same propulsion system during all flight modes. Additionally, this study is only concerned with aircraft that use only electric or reciprocating propulsion systems. Although dealing with any other type of aircraft or propulsion system separately is possible, it is considered to be practically not possible to encompass all types in one design methodology.

b. The proposed work centers on the design of TA by only focusing on the transition aspects from VTOL to fixed-wing.

c. The transition is constraint to only take place in straight horizontal level forward flight. Thus, we do not consider transition occurring in any other basic or acrobatic flight mode such as banks, turns, climb or descent, barrel roll, Cuban eight, etc.

d. The aircraft's transition is constraint to take place in the absence of turbulent flows existing in the atmosphere. That is, in this paper, we do not consider the effects of turbulent and cross flows such as cross wind gusts or wake flows caused by (for example) aircraft flying in front of the aircraft of interest.

e. The proposed method will be applicable to aircraft that have no or have a small wing area under the rotors during hovering.

\section{Proposed methodology}

In order to address and provide a solution to the above-mentioned problem(s) under the assumptions and constraints described in Section 4 , this paper proposes a preliminary design methodology for tiltrotor aircraft based on a three-step process.

a. Establishing a performance constraint during the transition phase 
of the aircraft. Such performance constraint relates the wing, disc and power loadings with the minimum required rotor tilt angle, which makes the aircraft wing-borne.

b. Enhance performance equations of rotorcraft systems to allow sizing the aircraft engine and rotor disc area according to the required rotorcraft performance.

c. Combine the fixed-wing preliminary design methodology 21 with rotorcraft while simultaneously integrating the transition performance requirements with the constraints into a single design chart to be used as a preliminary design methodology for TA.

The proposed design chart will include a three-axis relationship between power loading, wing loading, and rotor disc loading, where the horizontal axis will be the power loading, and the left/right vertical axis will be the wing loading and the rotor disc loading, respectively. This proposed three step process methodology comprise and takes into account the required three flying modes (helicopter, transition and fixed-wing) to generate a set of curves (chart) that will enable sizing the engine (power loading), wing area (wing loading), and the rotor disc area (disc loading) according to the required performance constraints during all flight modes. The suggested method is developed and targeted for aircraft that use the same engine for both fixed-wing and helicopter flying modes. Due to the associated challenges, emphasis is placed on the transition from helicopter to fixed-wing whereas relaxing the operational flight conditions during the transition from fixed-wing to helicopter mode (e.g., possibly allowing longer transition time). It is expected that with this proposed method, one can proceed to draw a set of curves that represent the required performance constraints in the three flight modes used in TA.

\section{Procedure for TA preliminary design}

As described in section 5, a mathematical approach is developed using variations of the typical mathematical formulations used in the independent design of fixed-wing aircraft and rotorcraft. Such formulations are then combined to find a meeting point where both types of aircraft merge targeting tiltrotor TA.

\section{A. Rotorcraft sizing}

Sizing of the aircraft in helicopter flight mode is performed based on traditional rotorcraft performance equations $\mathrm{s}^{22,23}$ used within the aerospace industry. It is well known, however, that there are a large number of performance requirements that contribute to engine power and rotor size. Example of such requirements include: the requirements for hovering at certain altitude, executing a vertical climb speed, hover at specific ceiling altitude, performing forward climb flight, performing some maneuvers, and descent flight. In order to simplify the process, the parameters that are expected to have the largest influence on the choice of the design point are first identified as suggested in, ${ }^{22,23}$ and then used in the proposed procedure. Thus far, the requirements for hovering flight, vertical climb flight, and ceiling altitude have been identified to have the higher power-to-weight ratio compared to forward climb flight and the descent flight. Additionally, such requirements are considered the most important in the choice of the corresponding rotorcraft disc loading. So, the performance equations of these rotorcraft flight regimes are selected to be used in the sizing calculations.
Generally, the total required power for rotorcraft to satisfy different performance requirements is given by the following equation: ${ }^{22,23}$

$$
P=\left(P_{i}+P_{o}+P_{p}+P_{c}+P_{T R}+P_{a c c}\right) \frac{1}{\eta_{t r}}
$$

where: $P_{i}$ is the induced power, $P_{o}$ is the profile power of the blades, $P$ is the parasite power required to overcome the drag of the airframe, $P_{c}^{p}$ is the climb power, $P_{T R}$ is the tail rotor power, $P_{a c c}$ is the power required to drive the accessory systems, and $\eta_{t r}$ is the transmission efficiency to account for power transmission losses.

In the proposed analysis for tiltrotor UAVs, the tail rotor power and the transmission loss will be omitted (e.g., battery operated vehicles and in dual tiltrotors as a tail rotor might not be needed). Additionally, depending on the flight mode, some of these power terms can be neglected if their values (effects on the aircraft) are very small compared to the others.

\section{Hovering flight}

During rotorcraft operation, a large number of transitional UAVs have the rotors over the wings that needed for fixed-wing operation (e.g., Rotormast transitional $\mathrm{UAV}^{24}$ ). This aspect complicates the design process, as extensive CFD has to be performed. However, if there is no wing area under the rotors (i.e., no download force is generated by the downwash airflow impacting the wing) as seen in a number of other TA (e.g., Quantum's Tron UAV) and by neglecting the parasite drag of the aircraft during hovering (as it is significantly small compared to aircraft's weight), the parasite power term, $P_{p}$, can be omitted. Under such conditions, the total power required for hovering is the sum of the induced, $P_{i}$, and the rotor profile power, $P_{o}$. This results in the need to only consider $P_{i}$ and $P$ for sizing the aircraft in hover flight.

From the one-dimensional axial momentum theory, ${ }^{22}$ the induced velocity at the rotor in hover, $v_{i}$, and the ideal induced power required to hover, $P_{i}$, are given by Eq. (2) where an additional parameter " $B$ ", typically found in aircraft formulations, is assumed to be equal to 1 (one):

$$
v_{i}=\sqrt{\frac{T}{2 \rho A}}, \quad P_{i}=T v_{i}=T \sqrt{\frac{T}{2 \rho A}}
$$

Where: $W$ is the aircraft's weight, $A$ is the rotor disk area, $T$ is the rotor thrust, and $\rho$ is the air density at the given hovering altitude.

By replacing the thrust with the weight and adding a correction factor to the induced velocity it is possible to include any rotor tip losses, non-uniform inflow, wake swirl, non-ideal wake contraction, and finite number of blades' effects on the aircraft. The obtained nonideal induced power equation is then given by:

$$
P_{i . \text { Nonideal }}=T v_{i . \text { Nonideal }}=T\left(k_{i} v_{i}\right)=k_{i} \frac{W^{3 / 2}}{\sqrt{2 \rho A}}
$$

where: the non-dimensional value $k_{i}$ is the induced power correction factor ${ }^{22}$ which has a typical average value in hovering of 1.15 .

The rotor profile power $\left(P_{o}\right)$, which is required to overcome the profile drag of the blades, is obtained by taking the drag of a blade element and integrating over the span of the blade (Eq. (4)). 


$$
P_{o}=\rho A V_{\text {Tip }}^{3}\left(\frac{\sigma C_{d}}{8}\right)
$$

In Eq. (4) $C_{d}$ is the average blade drag coefficient, $\sigma$ is the main rotor solidity ratio (ratio between total blades area and rotor disc area), and $V_{T i p}$ is the rotor's tip speed.

At this design stage, there are no precise data about a number of design parameters such as the number of rotors, rotor diameter, tip speed, airfoil type, solidity, drag, etc. which prevents the exact calculation of the power required for the rotor profile drag. To account for the power needed to overcome these effects, the Figure of Merit $(F o M)$ which is the ratio of ideal induced power for a rotor in hover obtained from momentum theory and the actual power consumed by the rotor (Eq. (5)), is used:

$$
F O M=\frac{P_{i}}{P}
$$

where by eliminating the suggested parameters from Eq. (1), the actual power consumed by the rotor in hover is given by Eq. (6).

$$
P=P_{i . \text { Nonideal }}+P_{o}=k_{i} \frac{W^{3 / 2}}{\sqrt{2 \rho A}}+\rho A V_{T i p}^{3}\left(\frac{\sigma C_{d}}{8}\right)=\frac{1}{F o M} \frac{W^{3 / 2}}{\sqrt{2 \rho A}}
$$

From Eq. (6), the relation between power and disc loading is obtained, Eq. (7) where $(P L=W / P)$ and $(D L=W / A)$ are the disc and power loadings, respectively.

$$
(P L)=F o M \sqrt{\frac{2 \rho}{(D L)}}
$$

The above equations are valid for configurations with no wing area under the rotor(s). If this is not the case, the assumption that the rotor thrust equals the weight is no longer valid. Therefore, an extra term should be added to the required power due to the vertical download force (vertical drag force) on the wing that results from rotor's downwash. This term varies from one aircraft configuration to another and depends on the equivalent drag area or drag coefficient per the wing to be used.

\section{Vertical climb flight}

The second aspect in the proposed rotorcraft design aimed towards $\mathrm{TA}$ is to consider the required vertical climb flight. As there is no asymmetric flow in purely vertical climb on the rotor blades, tiltrotors act as a single main rotor helicopter. Thus, the basic requirements for vertical climb can be calculated based on the axial momentum theory. ${ }^{22}$ For a Rate of Climb $(R o C), V_{y}$, the mass flow rate through the assumed single disk and the induced velocity are given by:

$$
\dot{m}=\rho A\left(V_{y}+v_{i}\right), \quad v_{i}=-\frac{1}{2} V_{y}+\frac{1}{2} \sqrt{V_{y}^{2}+\frac{2 T}{\rho A}}
$$

Assuming there is no download force on the wing and neglecting the parasite drag of the aircraft, the climb and induced power required in vertical climb can be formulated as indicated in Eq. (9):

$$
P_{c}+P_{i}=T\left(V_{y}+k_{i} v_{i}\right)=T V_{y}-\frac{k_{i}}{2} T V_{y}+\frac{k_{i} T}{2} \sqrt{V_{y}^{2}+\frac{2 T}{\rho A}}
$$

To account for the rotor's profile drag, and assuming there is no substantial change in profile power compared to hovering, the hovering profile power $(P)$ can be obtained as per Eq. (4). Then, the total power needed can be formulated as Eq. (10).

$$
P=P_{c}+P_{i}+P_{o}=\left(T V_{y}-\frac{k_{i}}{2} T V_{y}+\frac{k_{i} T}{2} \sqrt{V_{y}^{2}+\frac{2 T}{\rho A}}\right)+\rho A V_{T i p}^{3}\left(\frac{\sigma C_{d}}{8}\right)
$$

Similarly, assuming that the aircraft's drag during vertical climb is very small when compared to the aircraft's weigh, it can then be assumed that for a climb with constant speed $(V)$ the following relationship holds: $T \sim W$ (the required thrust is equals to the UAV's weight). Thus, reformulating Eq. (10), the relation between the required power and disc loadings during rotorcraft vertical climb is obtained (Eq. (11)).

$$
(P L)=\frac{1}{V_{y}-\frac{k_{i} V_{y}}{2}+\frac{k_{i}}{2} \sqrt{V_{y}^{2}+\frac{2(D L)}{\rho_{o}}}+\frac{\rho_{o} V_{T i p}^{3}}{(D L)}\left(\frac{\sigma C_{d}}{8}\right)}
$$

\section{Hover ceiling}

The rotorcraft's hover ceiling requirements is the last of the considered important aspects on TA design in the proposed approach. In this approach, however, the service ceiling will be used in the performance equations instead of the absolute ceiling to allow hovering flight with a stability margin at the desired ceiling altitude. A typical value for the $R o C$ at service ceiling for low subsonic aircraft is $V_{y . c}=0.5 \mathrm{~m} / \mathrm{s}^{22}$ Typically, for tiltrotor UAVs, either an electric motor or a reciprocating engine is used. To account for the reduction in the motor/engine power with altitude, the total power in Eq. (10) needs to be replaced with Eq. (12) where: $\sigma_{a i r}$ is the relative air density at the desired altitude (e.g., service ceiling).

$$
P_{\text {ceiling }}=P\left(\frac{\rho_{C}}{\rho_{o}}\right)=P \sigma_{\text {air }}
$$

Typical electric motors are designed to operate at sea-level ambient temperatures and below $1000 \mathrm{~m}$. For altitudes above $1000 \mathrm{~m}$, the output power is affected by the motor's ability to dissipate heat due to the reduction in the surrounding air density. To account for such reduction in motor's power, a factor called "de-rating factor", usually specified by the motor's manufacturer, is used at some specific altitude ranges above $1000 \mathrm{~m}$. In this paper, the symbol of $\sigma_{\text {air }}$ is also used to denote the de-rating factor if electric motor is used.

Substituting Eq. (12) in Eq. (11) to replace the power in the $(P L)$ term and use $R o C$ and density at service ceiling altitude, expression (13) is obtained which summarizes the relation between the power and disc loadings at the hover service ceiling altitude where: $\rho$ is the air density at the service ceiling altitude.

$$
(P L)=\frac{\sigma_{\text {air }}}{V_{y_{C}}-\frac{k_{i} V_{y_{C}}}{2}+\frac{k_{i}}{2} \sqrt{V_{y_{C}}^{2}+\frac{2(D L)}{\rho_{C}}}+\frac{\rho_{C} V_{T i p}^{3}}{(D L)}\left(\frac{C_{d} \sigma}{8}\right)}
$$

Now, the identified parameters for the rotorcraft that will led to a TA have been obtained. The next phase of the proposed design approach that formulates the UAV preliminary sizing according to the transition phase requirements can be developed.

\section{B. Transition flight sizing}

To formulate the equations necessary for aircraft sizing during the 
flight's transition mode, it is required to account for the following power terms: induced blades' profile, parasite, and climb. The climb term is only needed if and only if there is a change in the aircraft's altitude during transition. These terms are selected because it is known that each of these power terms has a considerable value at moderate forward flight speeds obtained during transition. ${ }^{22,23}$ Additionally, in tiltrotor aircraft, the sizing equations should include the required rotor tilt angle that makes the aircraft completely airborne by the lift generated by the wing. This angle should corresponds to the minimum forward flight velocity at which sufficient wing's lift is generated to carry the aircraft without any vertical thrust from the propellers. These parameters are similar to the parameters used in the rotorcraft sizing equations presented in Section A. Therefore, it was concluded that it is possible to formulate the aircraft sizing for the transition mode base on modifying the sizing equations in rotorcraft mode and include the rotor tilt angle rather than modifying the sizing equations in fixed-wing and have them meet the rotorcraft sizing in the middletransition phase (as an alternative approach). The reason for selecting this approach was due to the fact that it was identified to have more accurate calculation of the required power compared to omitting some power terms in fixed-wing approach (e.g., rotor profile power).

Assuming it is required to size the aircraft to perform the transition at a fixed altitude, the power required to climb is zero. Assuming level transition flight (flight path angle is zero), and for small angle of attack, the perpendicular component of forward flight velocity to the disc can be considered very small compared to the induced velocity at rotor disc. So, the induced velocity can be approximated by momentum theory as given in Eq. (14). According to the available rotorcraft forward flight performance equations,,$^{22,23}$ the non-ideal induced power term during transition flight can be formulated as given by Eq. (15). Whereas the power required to overcome rotor profile drag is formulated as in Eq. (16).

$$
\begin{gathered}
v_{i}=\sqrt{\frac{-V_{\infty}^{2}}{2}+\sqrt{\left(\frac{V_{\infty}^{2}}{2}\right)^{2}+\left(\frac{T}{2 \rho A}\right)^{2}}} \\
P_{\text {i.Nonideal }}=k_{i}\left(T v_{i}\right)=k_{i} T \sqrt{\frac{-V_{\infty}^{2}}{2}+\sqrt{\left(\frac{V_{\infty}^{2}}{2}\right)^{2}+\left(\frac{T}{2 \rho A}\right)^{2}}}
\end{gathered}
$$

$$
P_{o}=\rho A V_{T i p}^{3}\left(\frac{\sigma C_{d}}{8}\left(1+4.6 \mu^{2}\right)\right)
$$

where: $k_{i}$ in forward flight is of value 1.2 (somewhat higher than in hovering $)^{25}$ and $\mu$ is the forward dimensionless velocity in rotor disc plane (advance ratio) given by Eq. (17) where: $V_{\infty}$ is the forward flight velocity which makes the aircraft wing-borne and $\alpha$ is the angle of attack between the free stream velocity and the rotor disk.

$$
\mu=\frac{V_{\infty} \cos (\alpha)}{V_{\text {Tip }}}
$$

The required parasite power to overcome the aircraft's drag as it gains forward speed during the transition phase is:

$$
P_{p}=D V_{\infty}=0.5 \rho V_{\infty}^{3} C_{D} S
$$

Thus, per Eqs. (15), (16), and (18) under a level-flight transition (zero climb/descent), the total required power for transition flight is

$$
P=k_{i} T \sqrt{\frac{-V_{\infty}^{2}}{2}+\sqrt{\left(\frac{V_{\infty}^{2}}{2}\right)^{2}+\left(\frac{T}{2 \rho A}\right)^{2}}}+\rho A V_{T i p}^{3}\left(\frac{\sigma C_{d}}{8}\left(1+4.6 \mu^{2}\right)\right)+0.5 \rho V_{\infty}^{3} C_{D} S
$$

Neglecting the small vertical component of the rotor profile drag, the force equilibrium in the vertical direction during level-flight transition can be expressed as a thrust force per Eq. (20) where: $\theta_{\text {tilt }}$ is the rotor tilt angle measured counter clock-wise from TA longitudinal axis.

$$
T=\frac{W}{\sin \left(\theta_{\text {tilt }}\right)}
$$

To compensate for the power required to overcome the wing's induced drag corresponding to wing-borne velocity, the aircraft's drag coefficient is represented by:

$$
C_{D}=C_{D o}+K C_{L}^{2}=C_{D o}+\frac{4 K(W / S)^{2}}{\rho_{o}^{2} V_{\infty}^{4}}, \quad K=1 /(\pi . e . A R)
$$

Hence, reformulating Eq. (19), the relation between power loading, disc loading, and wing loading as a function of the rotor tilt angle and wing-borne forward speed can be deduced as follows:

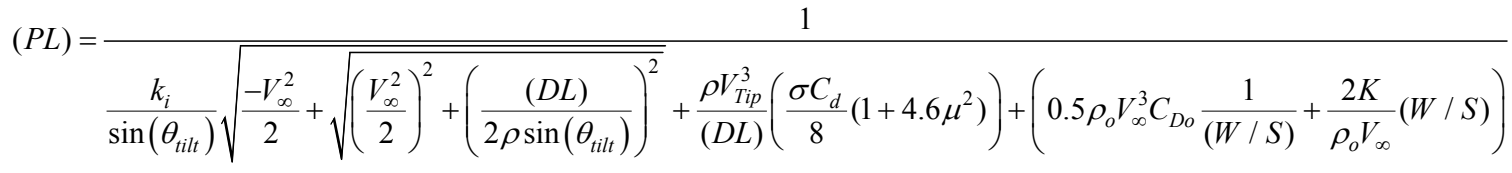

This approach provides an effective approach that enables plotting the variation of the power loading with the corresponding disc loading at different values of wing loading. Using the wing loading obtained from the fixed-wing performance formulations (described in Section C), Eq. (22) significantly aids in obtaining the proper engine and rotor sizes that makes the aircraft completely wing-borne at the required rotor tilt angle.

\section{Fixed-wing sizing}

The last flight phase to be considered for a TA is its operation in fixed-wing mode. Similarly to the approach used when sizing the aircraft in rotorcraft mode, classical performance equations of fixed-wing aircraft ${ }^{21}$ are herein used to allow obtaining a relationship function between wing loading and the corresponding power loading. The four performance requirements selected in this paper for sizing the aircraft in fixed-wing mode are:
a. The stall speed.
b. The maximum forward speed.
c. The maximum $R o C$.
d. The maximum altitude (ceiling). 
In addition, the requirement for the aircraft to perform both conventional and short runway Take-off must be considered and added to the approach as the aircraft might be required to perform such operation instead of VTOL to save power or be able to lift heavy loads, which might not be possible in VTOL.

\section{Stall speed}

Stall speed is one of the important fixed-wing aircraft performance requirements that limit the cruise speed to a minimum allowable value. It is important to consider such a limit in the design to prevent aircraft stall during its operation. As the aircraft's weight must be balanced with the lift during cruise flight, the wing loading can be formulated as a function of the stall speed as given in Eq. (23).

$$
(W / S)=0.5 \rho_{o} V_{s}^{2} C_{L \max }
$$

where: $W / S$ is the wing loading, $\rho_{o}$ is the air density at sea level, $V_{s}$ is the required stall speed and $C_{L \max }$ is the aircraft maximum lift coefficient.

\section{Maximum forward speed}

The second considered performance requirement is the maximum forward speed. Both wing and power loadings contribute in achieving this parameter. As our concern is tiltrotor aircraft, the performance equations of propeller-driven aircraft are used to formulate a relationship between the power loading as a function of the wing loading and maximum speed as in Eq. (24).

$$
\begin{gathered}
(W / P)=\frac{\eta_{p}}{0.5 \rho_{o} V_{\max }^{3} C_{D o} \frac{1}{(W / S)}+\frac{2 K}{\rho \sigma_{\text {air }} V_{\max }}(W / S)} \\
K=1 /(\pi . e . A R)
\end{gathered}
$$

where: $W / P$ is the power loading, $\eta_{p}$ is the propeller efficiency, $C_{D o} \quad$ is the aircraft zero lift drag coefficient, $\sigma_{\text {air }}$ is the relative air density/de-rating factor, $\rho$ is the air density at the altitude of maximum speed, $V_{\max }$ is the required maximum speed, $A R$ is the wing aspect ratio and $e$ is the Oswald efficiency.

\section{Maximum rate of climb}

Another performance requirement is to climb in fixed-wing flight mode by a certain $R o C$. Similarly, the climb performance equations of propeller-driven aircraft are formulated to give the relationship between the power loading as a function of the wing loading and $\mathrm{RoC}$ as in Eq. (25) where: $(L / D)_{\text {max }}$ is the maximum lift to drag ratio and $V_{y}$ is required rate of climb.

$$
(W / P)=\frac{1}{\frac{V_{y}}{\eta_{p}}+\left[\frac{1.155}{(L / D)_{\max } \eta_{p}}\right] \sqrt{\frac{2}{\rho_{o} \sqrt{3 C_{D o} / K}}(W / S)}}
$$

\section{Ceiling}

Ceiling in fixed-wing aircraft, the highest altitude that an aircraft can safely fly straight and level, is another requirement that affect wing and power loadings. In this formulation, we deal with the service ceiling instead of absolute ceiling to allow straight and level flight with a good stability margin at ceiling altitude. A typical value for the $R o C$ at service ceiling for low subsonic aircraft is $V_{v . c}=0.5 \mathrm{~m} / \mathrm{s}^{21}$ Since the ceiling requirements are defined based on the $R o C$ requirements, Eq. (25) is reformulated at service ceiling parameters as shown in Eq. (26) where: $V_{y . c}$ is the rate of climb at service ceiling and $\rho_{c}$ is the air density at service ceiling.

$$
(W / P)=\frac{\sigma_{\text {air }}}{\frac{V_{y \cdot \mathrm{c}}}{\eta_{p}}+\left[\frac{1.155}{(L / D)_{\max } \eta_{p}}\right] \sqrt{\frac{2}{\rho_{C} \sqrt{3 C_{D o} / K}}(W / S)}}
$$

\section{Short/Conventional take-off run}

The minimum take-off ground run distance is one of the important factors that affect the wing and power loadings. The take-off performance equations ${ }^{21}$ of propeller-driven fixed-wing aircraft are modified to include thrust vectoring for Short Take-off and then used to derive the relationship between the power loading as a function of the wing loading and take-off distance as in Eq. (27). This equation is a general one that can be used to size both short take-off and conventional take-off aircraft by either putting the thrust vector angle $(\theta)$ with certain value or zero, respectively.

$$
(W / P)=\frac{-\cos (\theta)-\mu_{f} \sin (\theta)+\left[\cos (\theta)+\mu_{f} \sin (\theta)+\left(C^{*} / C_{L_{R}}\right) \sin (\theta)\right] \cdot e^{\left(\rho g C^{*} S_{G} /(W / S)\right)}}{-\mu_{f}+\left(\mu_{f}+C^{*} / C_{L_{R}}\right) \cdot e^{\left(\rho g C^{*} S_{G} /(W / S)\right)}} \frac{\eta_{p}}{V_{T O}}
$$

$$
C^{*}=C_{D_{T O}}-\mu_{f} C_{L_{T O}}, C_{L_{R}}=\frac{2 m g}{\rho S V_{R}^{2}}, C_{D_{T O}}=C_{D_{o . T O}}+K C_{L_{T O}}^{2}
$$

In Eqs. (27) and (28) $\theta$ represents the rotor tilt angle measured from aircraft longitudinal axis, $\mu_{f}$ is the runway friction coefficient, $C_{\text {DTO }}$ is the drag coefficient during take-off, $C_{\text {ВотO }}$ is the zero-lift drag coefficient during take-off, $C_{L T O}$ is the lift coefficient during take-off, $C_{L R}$ is the lift coefficient at take-off rotation, $S_{G}$ is the total take-off ground run distance, $V_{R}$ is the aircraft speed at rotation usually it is 1.1 to 1.2 of the stall speed, and $V_{T O}$ is the take-off speed and usually it is 1. to 1.3 of the stall speed.

\section{Tiltrotor preliminary design chart}

By integrating all mathematical formulations developed in sections A, B, and C, a preliminary design chart for tiltrotor aircraft, that represent the required performance constraints in the three flight modes (helicopter, transition, and fixed-wing), can now be fully developed and the optimum preliminary design parameters can be selected. Figure 3 provides an example set of curves that illustrate the expected type of chart and results from the proposed design methodology. The proposed chart and its representation scheme provide the required variation of the power loading with respect to wing and disc loadings. In contrast to traditional aircraft design curves, the proposed chart includes regions for both fixed-wing and rotorcraft design, which will enable engineers to best select aircraft 
parameters and regions of flight performance. For fixed-wing mode, the variations of the wing loading with respect to the power loading are illustrated via the left vertical axis and the horizontal axis. On the other hand, the variations of the disc loading with respect to the power loading for the helicopter mode is extracted from the right vertical axis and the horizontal axis. For the transition mode, a curve (represented in red color in Figure 3) is drawn in the chart to illustrate the variations of the disc loading with the power loading at the value of wing loading obtained from the fixed-wing design point.

For each flight mode, the intersection of all the above-described curves generates a number of design regions. The feasible design region, for each flight mode, will be identified as the region in which the power and wing or rotor disc loadings meet all required performance constraints. For illustration purposes in Figure 3, in fixed-wing mode the "fixed-wing feasible design region" is outlined by the intersection of the areas to the left of all fixed-wing performance constraints (black curves) except for the stall speed limit (bold solid black line) where the feasible fixed-wing design region is to be located under this stall speed limit. The fixed-wing design region is shaded in gray in Figure 3. In a similar way, the "rotorcraft feasible design region" is formed by the intersection of the areas to the left of the helicopter performance constraints (orange curves). In Figure 3 such region is shaded by yellow color. For transition, the "Tiltrotor feasible design region" is formed by the intersection of the rotorcraft feasible design regions and the area to the left of the transition performance constraints (red curve).

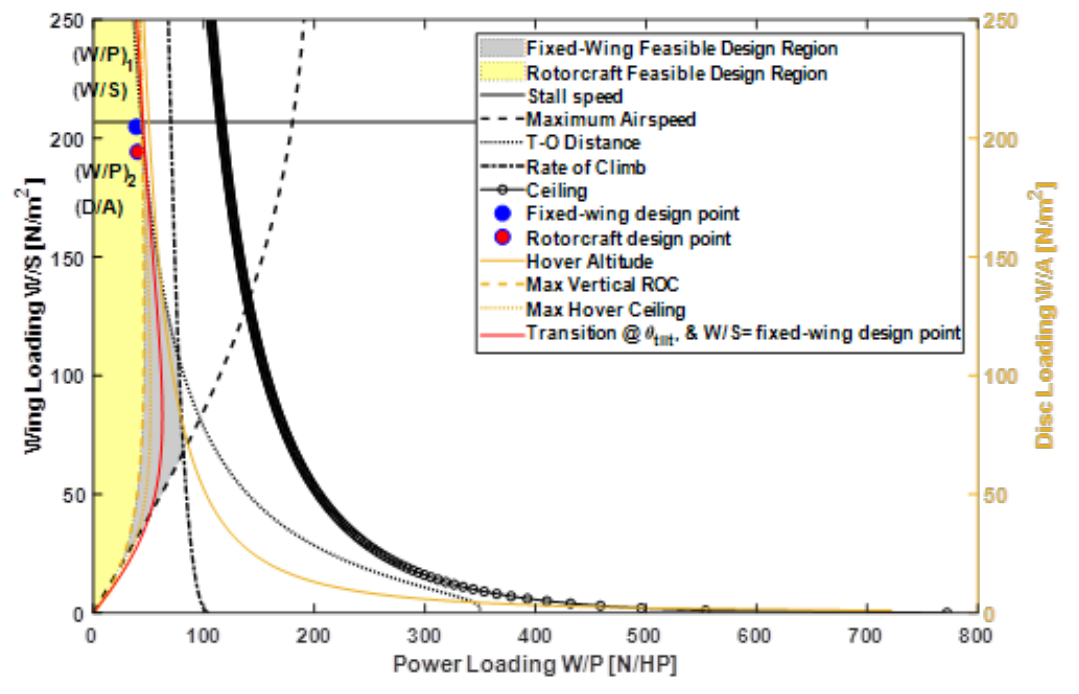

Figure 3 Typical example for the proposed preliminary design chart for tiltrotors.

Typically, the optimum preliminary design parameters for fixedwing aircraft are the maximum available power and wing loading, considering the power is more important, that satisfy all performance constraints. For selecting the optimum preliminary design parameters, two points are considered:

a. The point that achieves the maximum power and wing loadings (considering the power is more important) inside the intersection of fixed-wing and rotorcraft feasible design regions is selected. This point is called herein "Fixed-wing design point" and is shown in Figure 3 with a blue dot.

b. The point that achieves the maximum power and disc loadings (considering the power is more important) inside the tiltrotor feasible design region (according to the obtained maximum wing loading) is also considered.

This point is named "Rotorcraft design point" and shown in Figure 3 with a red dot. Finally, the tiltrotor optimum preliminary design parameters are the obtained wing and disc loadings and the minimum of the two obtained values of the power loadings. The procedure for selecting the tiltrotor's preliminary design parameters is illustrated by a flowchart in Figure 4. As a result from the proposed chart, one should be able to select the design parameters for the TA of interest. After selecting the design parameters, the aircraft power, wing area, and rotor diameter can be calculated using the estimated TA weight.

\section{Preliminary design of transitional tiltrotor UAV}

The goal of the proposed approach is to enable effective design for transitional tiltrotor UAVs having superior performance when compared to current developed TA. This paper however focuses on a highly maneuverable tiltrotor UAV that has been under development in Calgary and selected to be used as a test bench to apply the proposed developments as a case study. Preliminary work on such aircraft has focused on the rotorcraft design aspects and its control architecture. ${ }^{26-29}$ In what follows, preliminary results of its transitional tiltrotor design as proposed in this paper are presented. Generally, aircraft design process includes four major phases:
a. Conceptual design.
b. Preliminary design. 
c. Detail design.

d. Test \& evaluation.

The work performed thus far in the design of our targeted UAV follows the same process and the next subsections provide the design requirements, conceptual design result, and preliminary design results obtained.

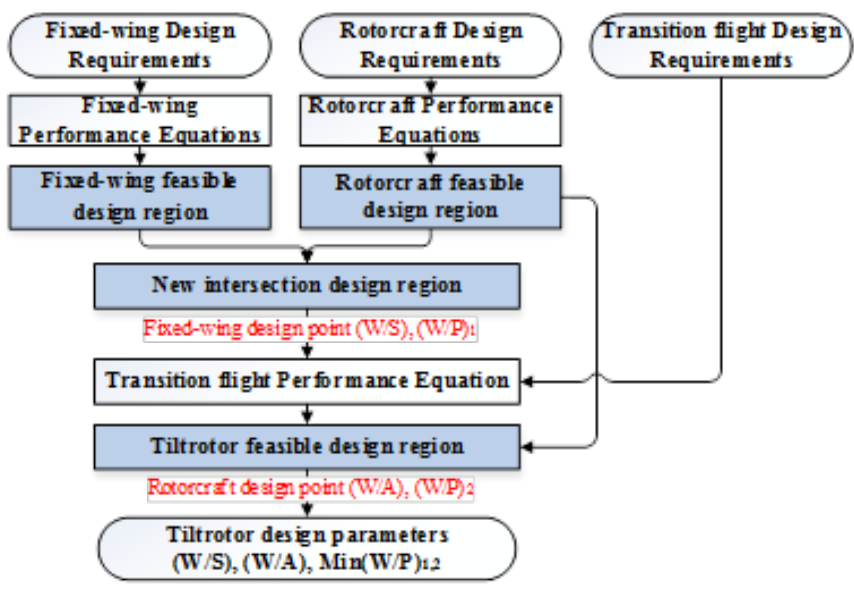

Figure 4 Selection process for the preliminary design parameters for tiltrotor aircraft.

\section{A. Design requirements}

The UAV design process was initiated by identifying the desired design requirements including the mission profile and required mission specifications. The envisioned goal is for the UAV to achieve high speed, enhance its operational range, while maintaining its current VTOL capabilities including its unique pitch hover and maneuver abilities in confined spaces.

\section{Mission profile}

A typical mission profile of the UAV can be described in a sequence of six steps as illustrated in Figure 5:

1) Take-off (either vertical or as a fixed-wing).

2) Hovering.

3) Transition from helicopter flight mode to fixed-wing flight mode.

4) Cruise as a fixed-wing UAV.

5) Transition from fixed-wing flight mode to helicopter flight mode.

6) Landing.

The above mission profile identifies the basic set of tasks that are aimed to be accomplished. Other specific tasks (e.g., enter a collapse building to search for potential victims) have not been included. Additionally, it must be noted that the mission profile does not explicitly includes several transition operations, as those are obvious and dependent on the aircraft's deployment goal(s).

\section{Mission specifications}

The required performance mission specifications in helicopter and fixed-wing modes have been selected as presented in Table 1.These performance mission specifications were selected as they illustrate typical desired specifications that will provide a good test bench to develop and analyze the proposed TA design methodology. With the selected mission specifications, one can move towards the first phase of the UAV design process, conceptual design. The next section presents the corresponding preliminary results.

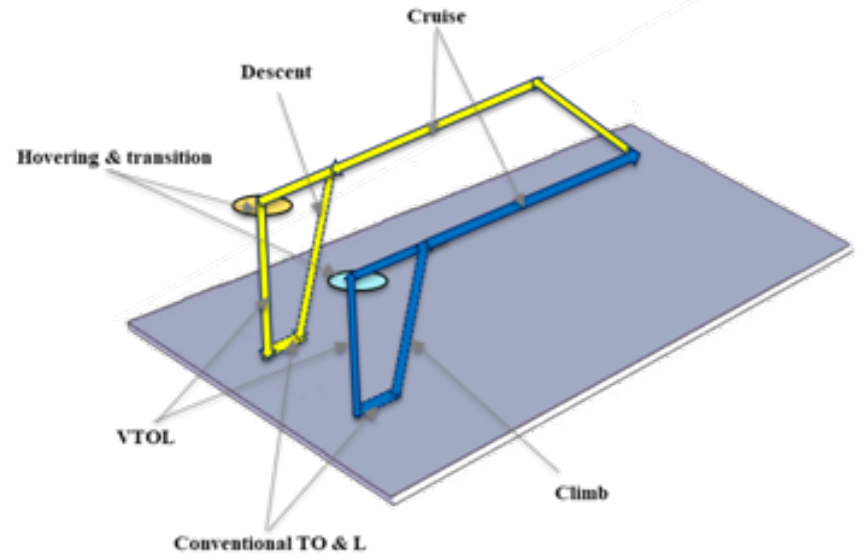

Figure 5 Mission profile of the transitional UAV.

\section{E. UAV conceptual design}

In selecting the aircraft's configuration, and to reduce the diverse complexities associated with transitional systems, three proposed configurations have been introduced and compared against each other. This step has been based on the available literature results, to identify the most suitable configuration. The selected configurations are:

1. Three lifting surfaces configuration with central ducted fans (Configuration 1).

2. Conventional wing-tail configuration with ducted fans at wingtips (Configuration 2).

3. Box-wing configuration with central ducted fan (Configuration $3)$.

Table I Summary of required mission specifications

\begin{tabular}{llll}
\hline Parameter & Value & Parameter & Value \\
\hline $\begin{array}{l}\text { Total Cruise Range } \\
{[\mathrm{km}]}\end{array}$ & 100 & $\begin{array}{l}\text { Fixed-wing rate of climb } \\
{[\mathrm{m} / \mathrm{s}]}\end{array}$ & 5 \\
$\begin{array}{l}\text { Cruise Altitude }[\mathrm{km}] \\
\text { Maximum Speed }\end{array}$ & 2 & $\begin{array}{l}\text { Runway length for take-off } \\
{[\mathrm{m}]}\end{array}$ & 30 \\
$\begin{array}{l}\text { [km/hr] } \\
\text { Stall Speed }[\mathrm{m} / \mathrm{s}]\end{array}$ & 15 & $\begin{array}{l}\text { Hovering altitude }[\mathrm{km}] \\
\text { Helicopter rate of climb } \\
{[\mathrm{m} / \mathrm{s}]}\end{array}$ & $\mathrm{I}$ \\
Payload weight $[\mathrm{kg}]$ & 2.3 & $\begin{array}{l}\text { Hover ceiling altitude }[\mathrm{km}] \\
\text { Hon }\end{array}$ & 2 \\
\hline
\end{tabular}

Based on the preliminary design of the three-tiltrotor aircraft configurations, the box-wing (Figure 6A) is selected as the best alternative to use in the proposed research. The wings of the selected aircraft are arranged in a similar geometrical configuration as AVY's transitional aircraft (Figure 6B) and uses two tilting rotors besides the fuselage. This design allows flying the aircraft in all modes (VTOL, transition, and fixed-wing) with practically one aircraft configuration, where most of the aircraft sub-systems are used in all flying modes. 
Thus minimizing the dead weight that is typically found used/added to existing TA in any flying mode. Additionally, the selection is based on the fact that this type of aircraft can provide a weight saving between $22 \%$ to $35 \%$ compared to equivalent aerodynamically conventional airplanes if its geometric parameters are well-designed. ${ }^{30}$ Moreover, this configuration provides a lower aircraft's drag due to the reduction/ elimination of the influence of the wingtip vortices. Also, the two wings allow designing an aircraft with reduced wingspan that would only require a light and simple internal structure. Thus, thinner wings can be used, which reduces the aircraft's profile drag. Another important aspect for TA is that box-wing aircraft are less prone to stalling if the wing design is optimized. This increases the UAV maneuverability especially at high angles of attack. Furthermore, using two wings allows obtaining low wing loading, which decreases the stall speed and consequently the transition time. Finally, the existence of the ducts between the two wings comprising a connected wing (or box wing) design diminishes the detrimental mutual aerodynamic interaction between the propellers and the wings.

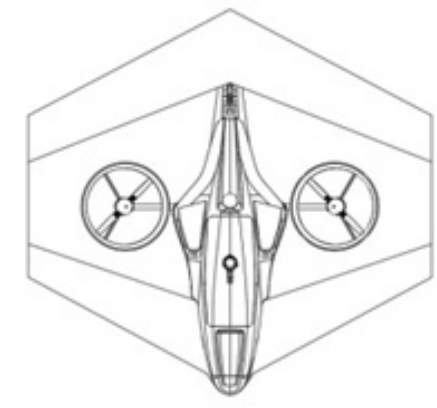

A) Selected box wing design

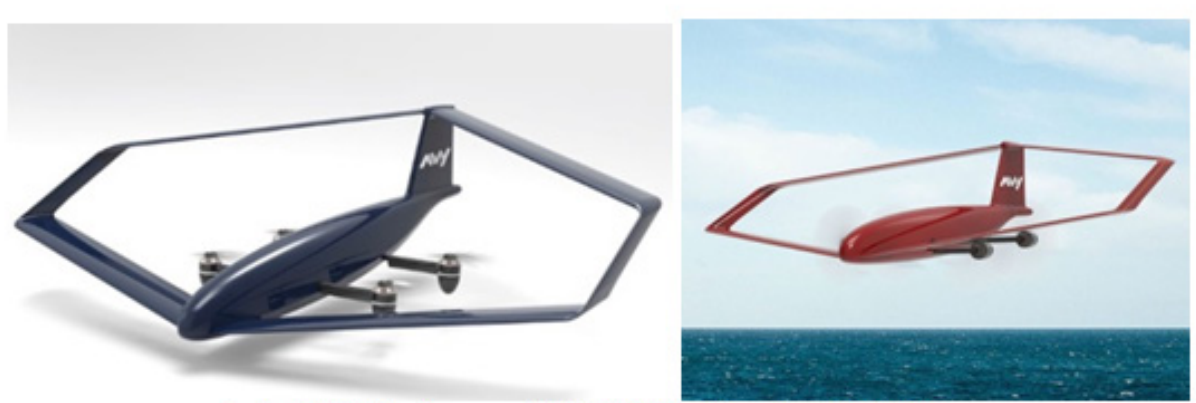

B) UAV from AVY (Netherlands) $)^{32}$

Figure 6 Illustrative visual layout for the proposed and concept box wing tiltrotor UAV.

In the selected configuration, all three propellers (two tilt ductedpropellers and one propeller at the end of the fuselage) will use a variable pitch system, which allow high maneuvers for the UAV and fast reaction time of the aircraft (e.g., to compensate for sudden wind gusts). The tri-propeller configuration is selected to help stabilize the UAV during hovering and transition. This configuration should also allow knotty and unique maneuvers like pitch hovering. The two tilt-propellers are selected to be ducted to increase thrust when compared to free propellers and increase cruise lift as they act as ring wings. The ducts position between the wings evades the detrimental aerodynamic interactions between the propellers and the wing, which should maximize hovering efficiency. The next section will include estimation to the UAV's weight (in the proposed box-wing configuration) and calculation of the power, wing, and disc loading resulting from the UAV preliminary design phase.

\section{F. Preliminary tiltrotor aircraft design}

This section includes the results of the selected transitional UAV preliminary design (initial sizing). These results include the estimation of take-off and empty weights, wing loading, power loading, rotor disc loading, wing area, wing aspect ratio, required Take-off power and maximum lift coefficient.

\section{Weight estimation}

The UAV maximum take-off weight and empty weight are estimated based on the weight build up method..$^{31}$ In order to calculate the empty weight fraction, ${ }^{31}$ a quick survey to similar fixed-wing UAVs (with a similar payload weight) was conducted and the results are presented in Figure 7. For the proposed aircraft configuration, the maximum take-off weight (for both VTOL and fixed-wing mode), $W_{T O}$, is found to be $15.351 \mathrm{~kg}$ with an empty weight, $W_{\text {Empty }}$, of $13.051 \mathrm{~kg}$.

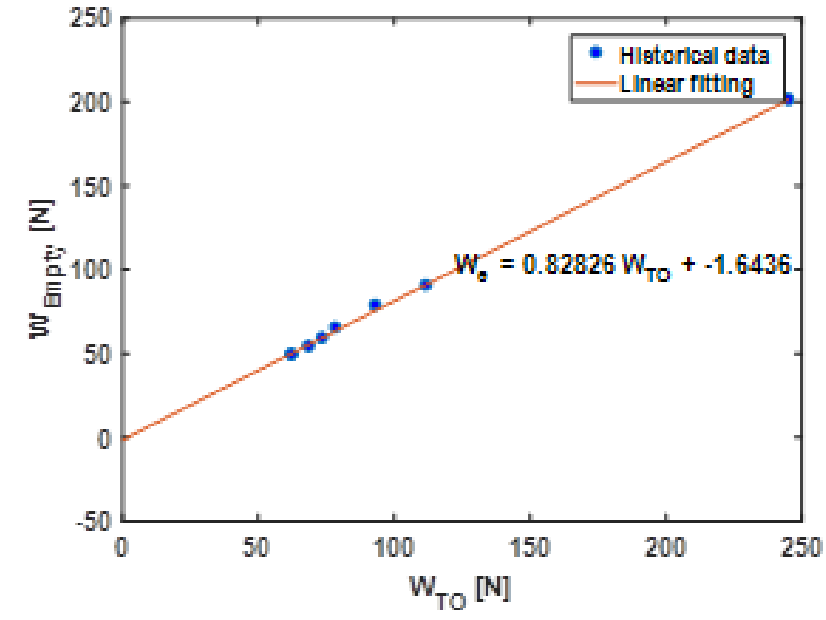

Figure 7 Relation between empty $\left(W_{\text {Empty }}\right)$ and take-off $\left(W_{\text {TO }}\right)$ weights for similar UAVs.

\section{Wing, rotor, and engine sizing}

Based on the mathematical formulations developed in Section 6, the proposed sizing constraint chart is plotted. The feasible design domains for the selected aircraft concept are identified and the optimum design parameters are considered as discussed in Section D. The values for the two identified parameter with their corresponding values are:

i) The fixed-wing design point is selected with a power loading value of $40.2 \mathrm{~N} / \mathrm{hp}$ and wing loading value of $204.77 \mathrm{~N} \backslash \mathrm{m}^{2}$. 
ii) The rotorcraft design point is selected (at rotor tilt angle equals 40 degree) with a power loading value $40.6 \mathrm{~N} / \mathrm{hp}$ and rotor disc loading value of $194.24 \mathrm{~N} \backslash \mathrm{m}^{2}$.

Finally, the optimum power loading is selected to be the minimum of the two values. Table 2 summarizes the obtained results of the TA preliminary design phase.

Table 2 Summary of UAV preliminary design phase

\begin{tabular}{|c|c|c|c|}
\hline Parameter & Value & Parameter & Value \\
\hline Take-off mass [kg] & $|5.35|$ & Empty weight [kg] & $|3.05|$ \\
\hline Wing loading $\left[\mathrm{N} / \mathrm{m}^{2}\right]$ & 204.77 & Power loading [NIhp] & 40.2 \\
\hline Wing area $\left[\mathrm{m}^{2}\right]$ & 0.735 & Wing aspect ratio [-] & 7 \\
\hline Wing span [m] & 2.268 & $\begin{array}{l}\text { Maximum take-off power } \\
\text { [hp] }\end{array}$ & 3.7 \\
\hline $\begin{array}{l}\text { Maximum lift } \\
\text { coefficient [-] }\end{array}$ & 1.5 & $\begin{array}{l}\text { Rotor disc loading [N1 } \\
\mathrm{m} 2]\end{array}$ & 194.24 \\
\hline
\end{tabular}

\section{Conclusion}

This paper presents a preliminary design methodology for transitional UAVs especially tiltrotor aircraft. By using the proposed mathematical formulations, it is possible to develop a comprehensive design methodology for TA by combining the performance constraints of the helicopter, transition, and the fixed-wing flight modes to develop a single design chart. This design chart enables selecting the optimum preliminary design parameters through obtaining the adequate values of the power, wing, and rotor disc loadings that are needed to satisfy the performance requirements in the three flying modes. This will help to avoid the unneeded excess power and consequently reduce the aircraft's weight and cost. Additionally, the proposed method helps in obtaining the power required to perform the transition flight at constant altitude. This will allow reducing the aircraft's transition time, and making the transition more smooth and stable. In addition, a proposed UAV configuration that shuns some of the frequent shortcomings of the previously developed types is presented. ${ }^{32}$

The proposed approach is a simple and straightforward methodology to generate an efficient graphical tool for selecting the most important preliminary sizing parameters for TA systems. By using the proposed mathematical formulations, the performance constraints of the FW, rotorcraft, and transition flight modes are integrated into a single tiltrotor design chart. This methodology enables rapid and manual selection of the most favorable preliminary sizing parameters without the complexities associated with the traditional numerical optimization methods. Moreover, the proposed approach surpasses the historical-data approach used in the design of numerous aircraft as it calculates the adequate power, wing, and rotor disc loadings based on the actual performance requirements in all flight modes. The validity of the developed methodology has been assessed by calculating the sizing parameters of the XV-15 aircraft and comparing the results with the actual numbers. The comparison reveals promising results as the errors do not exceed 3\%. Thus, the developed methodology is very helpful in rapid sizing of TR systems, with open and non-overlapping/ non-coaxial rotors, in all flight modes.

Future work will further validate the proposed methodology using data for other TR configurations. Additionally, the mathematical formulations may be extended to comprise tiltrotor aircraft with overlapping and coaxial rotors as well as tilt-wing and tail sitter configurations. Furthermore, additional performance constraints for maneuvering flights in rotorcraft mode may be added (e.g., turning maneuver).

\section{Acknowledgements}

None.

\section{Conflict of interest}

The author declares that there is no conflict of interest.

\section{References}

1. Ahn O, Kim J, Lim C. Smart UAV Research Program Status Update: Achievement of Tilt-Rotor Technology Development and Vision Ahead. 27th Congress of International Council of the Aeronautical Sciences; 2010. 8 p.

2. Bramlette RB, Barrett-Gonzalez RM. Design and Flight Testing of a Convertible Quadcopter for Maximum Flight Speed. USA: 55th AIAA Aerospace Sciences Meeting; 2017. 1-19 p.

3. Alli P, Nannoni F, Cicalè M. Erica: The European Tiltrotor Design and Critical Technology Projects. AIAA International Air and Space Symposium and Exposition: The Next 100 Years; 2003. 14-17 p.

4. The V/STOL Wheel. USA: Vertipedia; 2017.

5. Saeed AS, Younes AB, Islam $\mathrm{S}$, et al. A review on the platform design, dynamic modeling and control of hybrid UAVs. Colorado: International Conference on Unmanned Aircraft Systems (ICUAS); 2015. 806-815 p.

6. Rotormast V-22 Osprey Scale UAV. Texas: Rotormast; 2017.

7. NASA GL-10 Greased Lightning; 2014.

8. AeroVironment Quantix tail sitter UAV. California: Avinc; 2017.

9. ALTI Transition. Knysna: ALTI; 2017.

10. AV-1 Albatross VTOL Aircraft. Texas: DroneTech; 2017.

11. JUMP 20. California: Arcturus; 2017.

12. Armutcuoğlu O, Kavsaoğlu M, Tekinalp O. Tilt Duct Vertical Takeoff and Landing Uninhabited Aerial Vehicle Concept Design Study. Journal of Aircraft. 2004;41:215-223.

13. Ozdemir U, Aktas YO, Vuruskan A, et al. Design of a commercial hybrid VTOL UAV system. Journal of Intelligent and Robotic Systems: Theory and Applications. 2014;74(1-2):371-393.

14. Aktas YO, Ozdemir U, Dereli Y, et al. A low cost prototyping approach for design analysis and flight testing of the TURAC VTOL UAV. Florida: International Conference on Unmanned Aircraft Systems; 2014. 1029$1039 \mathrm{p}$.

15. Young LA, Lillie D, Yamauchi GK, et al. Insights into Airframe Aerodynamics and Rotor-on-Wing Interactions from a 0.25-Scale Tiltrotor Wind Tunnel Model. California: AHS International Aerodynamics, Acoustics, and Test and Evaluation Technical Specialists Meeting; 2002. 14 p.

16. Raymer D. Aircraft Design: A Conceptual Approach 5e and RDSWin STUDENT. Washington: American Institute of Aeronautics and Astronautics; 2012. $1044 \mathrm{p}$.

17. Keith JD, Hall DW. Rapid Sizing Methodologies for VTOL UAVs. Florida: 47th AIAA Aerospace Sciences Meeting Including the New Horizons Forum and Aerospace Exposition; 2009. 1-11 p. 
18. Roskam J. Airplane Design Part I : Preliminary Sizing of Airplanes. USA: DAR Corporation; 1985. 220 p.

19. Wang B, Hou Z, Liu Z, et al. Preliminary Design of a Small Unmanned Battery Powered Tailsitter. International Journal of Aerospace Engineering. 2016;3570581:1-11.

20. Holsten J, Ostermann T, Moormann D. Design and wind tunnel tests of a tiltwing UAV. CEAS Aeronautical Journal. 2011;2(1-4):69-79.

21. Sadraey MH. Aircraft design: A systems engineering approach. New Jersey: Wiley; 2012. 808 p.

22. Johnson W. Helicopter theory. New York: Dover Publications; 1980.

23. Filippone A. Flight Performance of Fixed and Rotary Wing Aircraft. UK: Butterworth-Heinemann; 2006. 600 p.

24. Do You Want To Convert? Texas: Rotormast.

25. Seddon J, Newman S. Basic Helicopter Aerodynamics. 3rd ed. USA: John Wiley \& Sons Ltd; 2011. 288 p.

26. Amiri N, Ramirez-Serrano A, Davies R. Modelling of Opposed Lateral and longitudinal Tilting dual-fan unmanned aerial vehicle. IFAC Proceedings Volumes. 2011;44(1):2054-2059.
27. Amiri N, Ramirez-Serrano A, Davies B. Nonlinear Adaptive Control of a New Configuration of Rotary Wing Unmanned Aerial Vehicle. Canada: 25th IEEE Canadian Conference on Electrical and Computer Engineering (CCECE); 2012.

28. Bagheri P, Ramirez-Serrano A, Pieper JK. Adaptive Nonlinear Robust Control of a Novel Unconventional Unmanned Aerial Vehicle. USA: Proceedings of the IASTED International Conference Intelligent Systems and Control; 2013. 7 p.

29. Majnoon M, Samsami K, Mehrandezh M, et al. Mobile-target tracking via highly-maneuverable VTOL UAVS with EO vision. Canada: Proceedings of 13th Conference on Computer and Robot Vision; 2016. 260-265 p.

30. Wolkovitch J. The joined wing-An overview. Journal of Aircraft. 1986;23:161-178.

31. Gundlach J. Designing Unmanned Aircraft Systems: A Comprehensive Approach. Reston: American Institute of Aeronautics and Astronautics; 2012. 805 p.

32. Avy boxed-wing UAV concept aircraft. Netherlands: Avy; 2017. 